Artigo original

Hegemonia - Revista Eletrônica do Programa de Mestrado em Direitos Humanos, Cidadania

e Violência/Ciência Política do Centro Universitário Unieuro

ISSN: $1809-1261$

UNIEURO, Brasília, número 25 (Especial), 2018, pp. 48-69.

Recebido em: 20/04/2018

Avaliado em: 25/04/2018

Aprovado em: 25/05/2018

\title{
A PRODUÇÃO CIENTÍFICA MUNDIAL DE ENSAIOS CLÍNICOS RANDOMIZADOS LIGADA À NANOTECNOLOGIA APLICADA À SAÚDE
}

\author{
Glécia Virgolino da Silva Luz, ${ }^{1}$ Lourdes Mattos Brasil, ${ }^{2}$ Breno Amadeus Sales Marinho de \\ Sousa, ${ }^{3}$ e Carleide dos Santos Moizinho ${ }^{4}$
}

Resumo: A manipulação do material em escala nanométrica tem impactado profundamente o desenvolvimento de novos produtos, em diversos setores. Recentes avanços indicam que a nanotecnologia representa uma promessa e esperança na solução de problemas, em áreas como engenharia e saúde. Quando tratada da área da saúde, os pesquisadores devem ultrapassar diversos estágios para design, síntese e testes de produtos. Como exemplo, citam-se os fármacos e biomateriais, que visam o diagnóstico e/ou tratamento de doenças, regeneração de tecidos, entre outros. Uma das etapas para realização de estudos experimentais pode-se aplicar a metodologia de Ensaios Clínicos Randomizados (ECRs), por serem considerados o mais confiável método para avaliar a eficácia de um tratamento ou intervenção. Assim, o objetivo deste trabalho é mostrar, a partir de buscas em bases de dados, a produção científica mundial de ECRs ligada à nanotecnologia aplicada à saúde. Para tanto, foram realizadas buscas de publicações nos últimos 10 anos, e com palavras-chaves em língua inglesa. Como resultado, observou-se que a produção relacionada à ECRs e saúde têm crescido substancialmente, sendo que, nos períodos de 2008 a 2012 e 2013 a 2018, houve um aumento de publicações em cerca de $35 \%$. Dessa forma, verificou-se que nos últimos 20 anos notou-se um expressivo aumento em área pesquisas sobre o tema abordado, ocasionando avanços significativos às áreas da saúde e nanotecnologia.

Palavras-chave: Nanotecnologia, Saúde, Intervenção, Ensaio Clínico Randomizado.

Abstract: Nanomaterial manipulation of material has profoundly impacted the development of new products in many industries. Recent advances indicate that nanotechnology represents a

\footnotetext{
${ }^{1}$ Doutora em Engenharia Mecânica e Pesquisadora Colaboradora do Programa de Pós-Graduação em Engenharia Biomédica da Universidade de Brasília, Faculdade Gama-FGA. Pesquisadora do Laboratório de Nanotecnologia NANOTEC-FGA/UnB.

2 Doutora em Engenharia Elétrica/Sistemas de Informação - Engenharia Biomédica, e docente na Universidade de Brasília. Coordenadora do Laboratório de Nanotecnologia - NANOTEC-FGA/UnB.

${ }^{3}$ Graduando em Engenharia de Energia da Universidade de Brasília.

${ }^{4}$ Mestranda no Programa de Pós-Graduação em Engenharia Biomédica da Universidade de Brasília.
} 
Artigo original

Hegemonia - Revista Eletrônica do Programa de Mestrado em Direitos Humanos, Cidadania

e Violência/Ciência Política do Centro Universitário Unieuro

ISSN: 1809-1261

UNIEURO, Brasília, número 25 (Especial), 2018, pp. 48-69.

promise and hope in solving problems, in areas such as engineering and health. When it comes to health care, researchers must go beyond several stages for product design, synthesis, and testing. As an example, mention is made of the drugs and biomaterials, which aim at the diagnosis and / or treatment of diseases, tissue regeneration, among others. One of the steps to perform experimental studies can be applied the methodology of Randomized Clinical Trials (RCTs), because they are considered the most reliable method to evaluate the effectiveness of a treatment or intervention. Thus, the objective of this work is to show, from database searches, the world scientific production of ECRs related to nanotechnology applied to health. In order to do so, we have been searching for publications in the last 10 years, and with keywords in English. As a result, it has been observed that production related to ECRs and health have increased substantially, and in the periods 2008 to 2012 and 2013 to 2018, there was an increase in publications by about $35 \%$. Hence, it has been observed that in the last 20 years there has been a significant increase in the area of research on the topic addressed, leading to significant advances in the areas of health and nanotechnology.

Keywords: Nanotechnology, Health, Intervention, Randomized Clinical Trial.

INTRODUÇÃO

A Nanotecnologia é hoje definida, segundo a National Nanotechnology Initiative (NNI, 2018), como a manipulação e controle da matéria de dimensões aproximadamente entre 1 e 100 nanômetros (nm). Nestas dimensões, fenômenos específicos permitem novas aplicações, as quais não estão presentes, ou são não viáveis, quando se trabalha com materiais a granel ou mesmo com átomos individuais ou moléculas (DEUS, 2014).

Observa-se uma tendência tecnológica impressionante que tem se destacada nas últimas décadas, pelos pesquisadores, e passa pelo rápido crescimento da eletrônica para aplicações em comunicação, saúde (chamada nanomedicina) e monitoramento ambiental. Atualmente, muitas pesquisas estão focadas nos gargalos científicos que permeiam a longevidade dos organismos vivos, especialmente os seres humanos (BARBOSA et al., 2015; LUZ et al., 2016). Pesquisas mostram que esta área tem trazido grandes descobertas, como nos setores de materiais, manufatura, eletrônicos, medicina, energia, biotecnologia, tecnologia da informação, segurança nacional, entre outros (BASTOS, 2006).

Há expectativas que esta tecnologia tenha um profundo impacto na economia e na sociedade atual, contrapondo à tecnologia da informação ou aos avanços na biologia celular e molecular. Observam-se que os principais destaques da nanotecnologia têm sido em três grandes áreas: materiais, energética, sistemas médicos e de saúde. A nanotecnologia, hoje, é uma indústria que movimenta trilhões de dólares. Deste montante, a área de materiais é responsável 
Artigo original

Hegemonia - Revista Eletrônica do Programa de Mestrado em Direitos Humanos, Cidadania e Violência/Ciência Política do Centro Universitário Unieuro

ISSN: 1809-1261

UNIEURO, Brasília, número 25 (Especial), 2018, pp. 48-69.

por cerca de 30\%, mas ainda existem outros mercados que são movimentados como: o campo da eletrônica, indústria farmacêutica, manufatura química, aeroespacial, etc. (WILLEMS; VAN DEN WILDENBERG, 2004).

Quando se vincula a nanotecnologia à área de saúde, verificam-se, cada vez mais, resultados promissores para a ciência e à sociedade como um todo. Ao se realizar buscas nas bases de dados de publicações científicas, observa-se a constante evolução em estudos que visam uma melhor qualidade de vida das pessoas. Estes estudos trabalham, por exemplo, na aplicação em engenharia de tecidos; diagnóstico ultrassensível; e em sistemas conhecidos como drug delivery (transporte, armazenamento e liberação de drogas); entre outros (SCHAFFAZICK et al., 2003).

Quando se trata de sistemas drug delivery, normalmente fala-se de nanopartículas que, devido ao seu tamanho, conseguem alcançar a célula alvo de uma forma perdurável. Uma das explicações para este fato, relaciona-se com a Elevada Permeabilidade e Retenção (EPR) dos vasos sanguíneos, resultando na relativa facilidade de passagem de macromoléculas ou nanopartículas. (SCHAFFAZICK et al., 2003).

Estudos que reúnem dados conjuntos nanotecnologia-saúde, passam por diferentes estágios, como: testes in vitro, in vivo, cada uma fase desses testes tem sua importância para o produto final. Tais procedimentos visam melhores resultados e caracterização do seu produto, passando por normas e comitês de ética (MALAVOLTA, 2011).

Assim, as fases das pesquisas clínicas se referem à sequência de estudos necessários para levantar informações se um tratamento é eficiente. O estágio de Ensaios Clínicos Randomizados (ECRs) são importantes para a segurança, qualidade, eficiência e eficácia de curto e longo prazo (MALAVOLTA, 2011).

Os ECRs são estudos experimentais, aplicados em seres humanos, e têm como objetivo o conhecimento dos efeitos de um determinado produto na intervenção da saúde do paciente avaliado. Estes estudos podem ser considerados como um amplo instrumento para obtenção de evidências para a prática clínica (BRASIL, 2012; COOK et al., 1992; SOUZA, 2009).

Dessa forma, produtos oriundos de estudos na escala nanométrica, visando a intervenção na saúde de um paciente, também devem seguir as fases que as pesquisas clínicas exigem. Existem inúmeros projetos de nanotecnologia com a aplicação a saúde, como nanoestruturas ou nanorobôs que são capazes de operar cada célula singularmente, podendo 
Artigo original

Hegemonia - Revista Eletrônica do Programa de Mestrado em Direitos Humanos, Cidadania e Violência/Ciência Política do Centro Universitário Unieuro

ISSN: 1809-1261

UNIEURO, Brasília, número 25 (Especial), 2018, pp. 48-69.

beneficiar pacientes com doenças crônicas, como o câncer. Supõe-se, também, que estes produtos serão menos tóxicos que os utilizados na medicina atual (BASTOS, 2006).

Assim, o objetivo deste trabalho é apresentar a produção científica mundial de ECRs ligada à nanotecnologia aplicada à saúde utilizando, para isso, pesquisas em bases de dados científicas, e em outros meios de divulgação de informações.

\section{METODOLOGIA}

Para o levantamento de dados sobre a produção científica mundial de ensaios clínicos randomizados ligada à nanotecnologia aplicada à saúde realizaram-se buscas em diferentes bases de dados científicos como: Science Direct, Lilacs, Web of Science, Periódico CAPES, PUBMED e Scielo. O período estipulado foi para os últimos 10 anos, isto é, de 2008 a 2018. De posse dos trabalhos elencados nas buscas, selecionou-se os artigos que contribuíssem com informações sobre produtos que estivessem em fase de estudos clínicos, neste caso, ensaios clínicos randomizados. Se não houvesse este tipo de informação, este seria excluído da seleção. As palavras-chaves/descritores, incluindo suas respectivas strings de busca, foram dispostos na Tabela 1.

Tabela 1. Strings utilizadas para as pesquisas nas bases de dados.

\begin{tabular}{|c|l|}
\hline Base de dados & \multicolumn{1}{|c|}{ String utilizada na busca } \\
\hline Science Direct & $\begin{array}{l}((\text { Nanotechnolog*) AND Health) AND (Treatment OR intervention OR diagnostic } \\
\text { OR therapy) }) \text { AND ("Randomized Controlled Trial" OR ("Clinical Trials" AND } \\
\text { Randomized) OR (Trials AND "Randomized Clinical")) }\end{array}$ \\
\hline Web of Science & $\begin{array}{l}\text { TS=(Nanotechnology AND Health AND (Treatment OR intervention OR diagnostic } \\
\text { OR therapy) AND ("Randomized Controlled Trial" OR ("Clinical Trials" AND } \\
\text { Randomized) OR (Trials AND "Randomized Clinical") OR ("Controlled Clinical } \\
\text { Trials" AND Randomized) OR "Randomized Clinical Trial")) }\end{array}$ \\
\hline Periódicos CAPES & $\begin{array}{l}\text { (Nanotechnolog*) AND Health AND (Treatment OR intervention OR diagnostic OR } \\
\text { therapy) AND ("Randomized Controlled Trial" OR ("Clinical Trials" AND } \\
\text { Randomized) OR (Trials AND "Randomized Clinical") OR ("Controlled Clinical } \\
\text { Trials" AND Randomized) OR "Randomized Clinical Trial") }\end{array}$ \\
\hline
\end{tabular}


Artigo original

Hegemonia - Revista Eletrônica do Programa de Mestrado em Direitos Humanos, Cidadania e Violência/Ciência Política do Centro Universitário Unieuro

ISSN: 1809-1261

UNIEURO, Brasília, número 25 (Especial), 2018, pp. 48-69.

\begin{tabular}{|c|l|}
\hline \multicolumn{1}{|c|}{ Lilacs } & $\begin{array}{l}\text { (tw:(Nanotechnolog*) }) \text { AND (tw:(Health)) AND (tw:(Treatment OR intervention OR } \\
\text { diagnostic OR therapy) }) \text { AND (tw:("Randomized Controlled Trial" OR ("Clinical } \\
\text { Trials" AND Randomized) OR (Trials AND "Randomized Clinical") OR ("Controlled } \\
\text { Clinical Trials" AND Randomized) OR "Randomized Clinical Trial")) }\end{array}$ \\
\hline Pubmed & $\begin{array}{l}\text { ((Nanotechnolog*) AND Health) AND (Treatment OR intervention OR diagnostic } \\
\text { OR therapy) }) \text { AND ("Randomized Controlled Trial" OR ("Clinical Trials" AND } \\
\text { Randomized) OR (Trials AND "Randomized Clinical")) }\end{array}$ \\
\hline Scielo & $\begin{array}{l}\text { (Nanotechnolog*) AND (Health) AND (Treatment OR intervention OR diagnostic } \\
\text { OR therapy) }) \text { AND ("Randomized Controlled Trial" OR ("Clinical Trials" AND } \\
\text { Randomized) OR (Trials AND "Randomized Clinical") OR ("Controlled Clinical } \\
\text { Trials" AND Randomized) OR "Randomized Clinical Trial") }\end{array}$ \\
\hline
\end{tabular}

\section{RESULTADOS}

Estudos relacionados às palavras-chaves/descritores utilizadas por esta pesquisa, revelaram um interessante crescimento. Segundo a Figura 1, percebe-se a presença de uma maior quantidade de trabalhos científicos, ligados aos assuntos de interesse, nas bases do site "Periódicos CAPES", e em seguida na Science Direct. Em números, os trabalhos relacionados foram 1758 e 805, respectivamente. Nas demais bases pesquisadas não foram encontradas publicações ou houve um número bem menor de resultados. 
Artigo original

Hegemonia - Revista Eletrônica do Programa de Mestrado em Direitos Humanos, Cidadania e Violência/Ciência Política do Centro Universitário Unieuro

ISSN: $1809-1261$

UNIEURO, Brasília, número 25 (Especial), 2018, pp. 48-69.

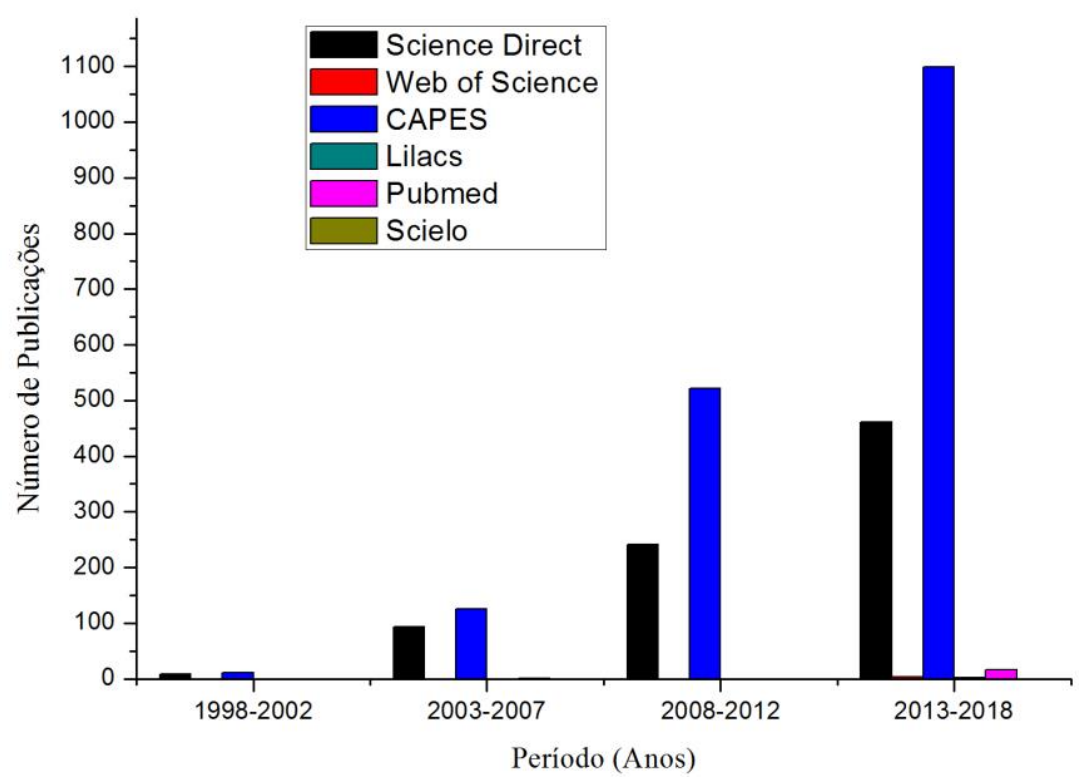

Figura 1. Gráfico contendo número de publicações em períodos de 5 em 5 anos, segundo dados fornecidos por pesquisas em diferentes bases de dados científicos. Fonte: Science Direct, Lilacs, Web of Science, Periódico CAPES, PUBMED e Scielo (Acesso em: abril de 2018) 
Artigo original

Hegemonia - Revista Eletrônica do Programa de Mestrado em Direitos Humanos, Cidadania e Violência/Ciência Política do Centro Universitário Unieuro

ISSN: $1809-1261$

UNIEURO, Brasília, número 25 (Especial), 2018, pp. 48-69.

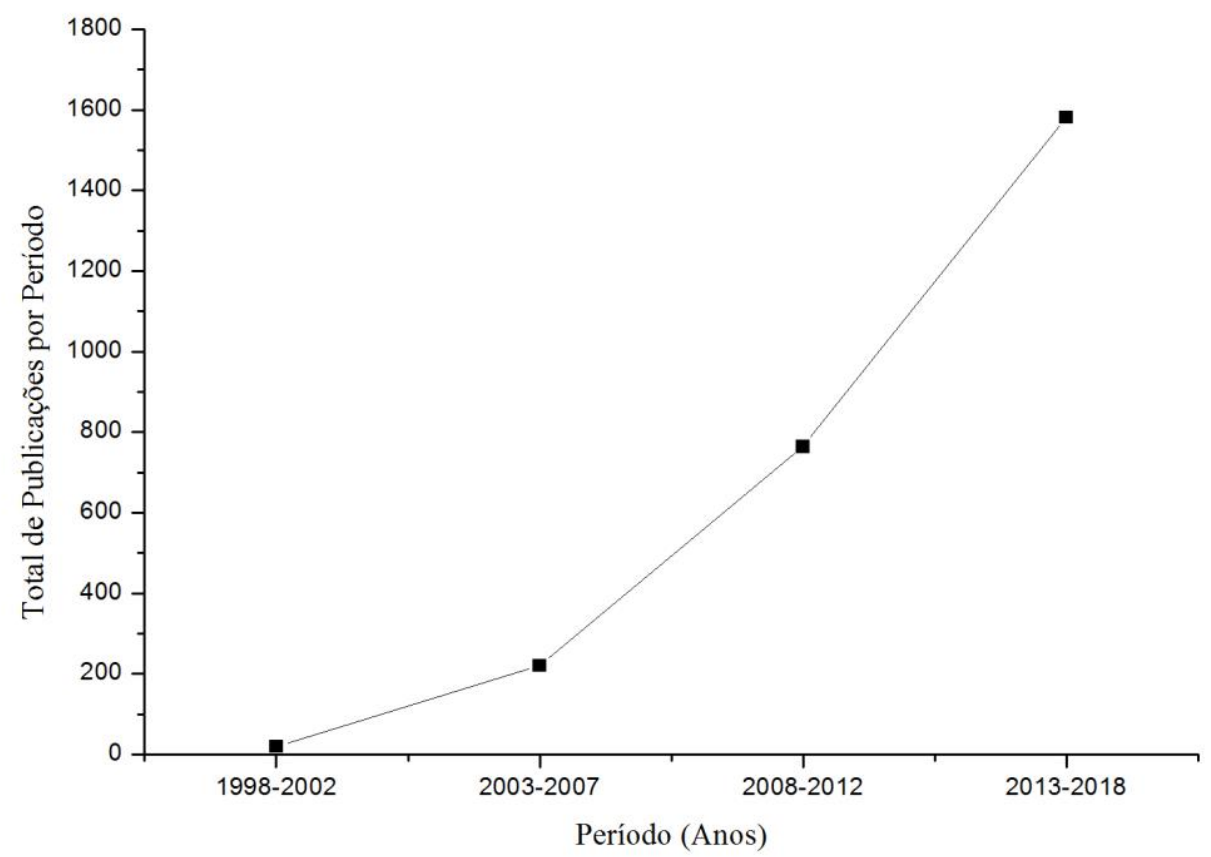

Figura 2. Gráfico contendo total de publicações em períodos de 5 em 5 anos, segundo dados fornecidos por pesquisas em diferentes bases de dados científicos. Fonte: Science Direct, Lilacs, Web of Science, Periódico CAPES, PUBMED e Scielo (Acesso em: abril de 2018).

Somente nos períodos de 2008 a 2012 e 2013 a 2018, houve um crescimento na quantidade de publicações em 35\%, isto é, passando de 764 a 1581 publicações a cada 5 anos (ver Figuras 1 e 2). A partir destes dados, percebe-se que nos últimos 20 anos (Figura 2) houve um crescente interesse pelo tema, confirmando-se o avanço na área de nanotecnologia e saúde. 
Artigo original

Hegemonia - Revista Eletrônica do Programa de Mestrado em Direitos Humanos, Cidadania

e Violência/Ciência Política do Centro Universitário Unieuro

ISSN: $1809-1261$

UNIEURO, Brasília, número 25 (Especial), 2018, pp. 48-69.

DISCUSSÃO

A PRODUÇÃO NANOTECNOLÓGICA MUNDIAL DE ECRs TESTADAS EM HUMANOS

São muitas as contribuições da nanotecnologia para a saúde e, particularmente, na pesquisa clínica da área biomédica. Algumas pesquisas discutem sobre os benefícios destas tecnologias no diagnóstico e/ou tratamento doenças como: diabetes, câncer, neurodegenerativas, pulmonares, mal de Alzheirmer, Parkison, cardiovasculares, controle de apetite, intervenções ortopédicas, terapia hormonal, equipamentos médicos, substitutos químicos, etc (CLINICALTRIALS.GOV, 2018; SAHA, 2009).

Especificamente nesta pesquisa, pôde-se encontrar artigos com os descritores préestabelecidos, como: diabetes gestacional, consumo de álcool, cardiopatias, aparelho respiratórios dentre outras, publicados no período de 2006 à 2017. Em se tratando de publicações de ensaios clínicos com nanomedicamentos, foram encontradas publicações ligadas, principalmente, a cárie dental, hipersensibilidade dental, menopausa (CLINICALTRIALS.GOV, 2018).

Nas indústrias farmacêuticas, o maior uso da nanotecnologia está na produção de fármacos voltados ao tratamento cancerígeno, que é denominada de nanosistema, o procedimento patológico para tratamento da doença acoplados na sua superfície ligantes que podem interagir com receptores que são expressos em células tumorais e associado aos nanosistemas o fármaco antitumoral (SCHAFFAZICK et al., 2003). Em geral, os sistemas de nanoestruturas mais usuais podem ser classificados, como: nanocápsulas, nanoesferas, nanopartículas, nanoemulsões, entre outros (SILVA, 2018).

O setor dos cosméticos também está ganhando o seu espaço com a implementação desta nova tecnologia, frente à sua característica de fácil absorção de inovações ágeis. Pode-se citar como resultados, produtos com fragrâncias e cosméticos mais eficazes. É importante destacar que a biodiversidade brasileira favorece, consideravelmente, a demanda de desenvolvimento de novas linhas de cosméticos, as quais possuem grande aceitação no mercado internacional, principalmente, quando se trata de higiene e cuidados estéticos (ANVISA, 2000). 
Artigo original

Hegemonia - Revista Eletrônica do Programa de Mestrado em Direitos Humanos, Cidadania e Violência/Ciência Política do Centro Universitário Unieuro

ISSN: 1809-1261

UNIEURO, Brasília, número 25 (Especial), 2018, pp. 48-69.

De acordo com as pesquisas bibliográficas sobre o tema proposto por esta pesquisa, constatou-se que, na indústria de cosméticos, a nanotecnologia possui aplicações em produtos de higiene. Como já relatado, para que o produto seja levado ao mercado, é necessário passar por estudos clínicos. A Figura 3a apresenta as regiões com o maior crescimento na Indústria dos Cosméticos no Brasil. Este crescimento segue a ordem crescente de: Sudeste $>$ Nordeste $>$ Centro Oeste. Este último vem ganhando o seu espaço no mercado internacional e tende a crescer cada vez mais, beneficiando a economia do país (GARCIA, 2005).

A Figura 3b apresenta os lugares do mundo onde a Indústria de Cosméticos está em pleno desenvolvimento e com isso, pode-se correlacionar os países que, consequentemente, possuem grande volume de ensaios clínicos e patenteamentos, realizados sobre produtos como os de higiene pessoal, perfumaria, entre outros.

Figura 3. Gráficos contendo dados sobre as Regiões e Países com o maior crescimento da Indústria dos Cosméticos. (a) Gráfico com informações sobre as Regiões do Brasil; e (b) Gráfico representando o comércio Mundial da Indústria de Cosméticos.
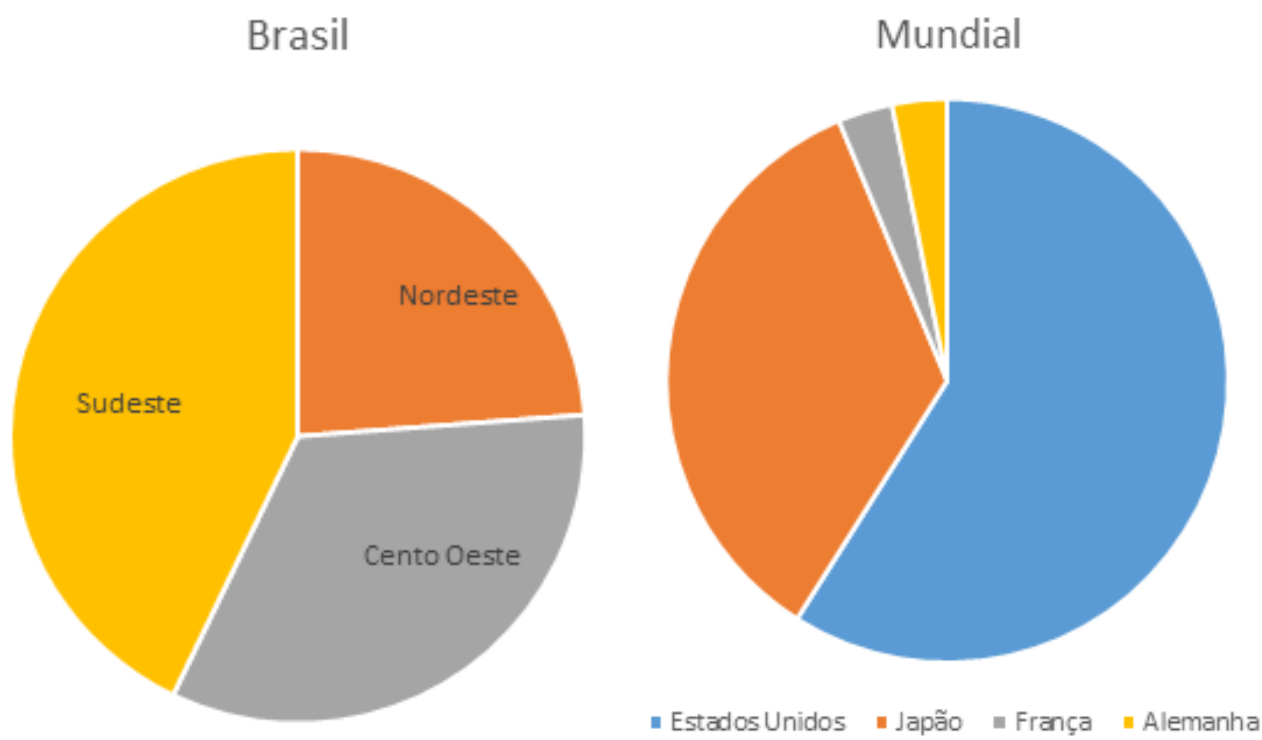

Fonte: GARCIA (2005). 
Artigo original

Hegemonia - Revista Eletrônica do Programa de Mestrado em Direitos Humanos, Cidadania e Violência/Ciência Política do Centro Universitário Unieuro

ISSN: 1809-1261

UNIEURO, Brasília, número 25 (Especial), 2018, pp. 48-69.

Com relação a patentes, dentre os países com melhores instituições para tal estão os Estados Unidos da América, seguido da França. O Brasil ainda tem morosidade no processo de patenteamento, segundo o Instituto Nacional de Propriedade Industrial (INPI) (BRASIL, 2017).

No Brasil, leva-se cerca de 30 meses para uma marca ser avaliada. Quando esta avaliação é para uma patente, esse prazo é de quase 11 anos, fato este que prejudica a capacidade de competição nacional em propostas de inovações industriais. De acordo com o INPI, o maior déficit de avaliação no Brasil está na área de biotecnologia e fármacos. Nos EUA esse tempo é de apenas dois anos (BRASIL, 2017).

Segundo a "U.S. National Institutes of Health", foram realizadas 207 pesquisas em pacientes com produtos que possuíam o termo "nanoparticle OR nanostructure", contendo ou não resultados e relacionados a intervenções (testes clínicos), sem especificação de data. Os status das pesquisas seriam: Ainda não recrutando, recrutando, se inscrevendo por convite, ativo, não recrutando e concluído. As pesquisas encontradas foram separadas em categoria de fármacos para: Agentes Anti-Infecciosos, Anticoagulantes, Antineoplásicos, Substitutos Sanguíneos, Agentes Fibrinolíticos, Micronutrientes, Doadores de Óxido Nítrico, Soluções Farmacêuticas, Agentes Vasodilatadores, entre outros. A Figura 4 apresenta as respectivas regiões envolvidas nestes testes (CLINICALTRIALS.GOV, 2018). Destes resultados, o Brasil contribui com 5 estudos.

Figura 4. Regiões envolvidas em 207 pesquisas clínicas com produtos que possuíam o termo "nanoparticle OR nanostructure", contendo ou não resultados e relacionados a intervenções

(testes clínicos), sem especificação de data, e segundo critérios de status específicos

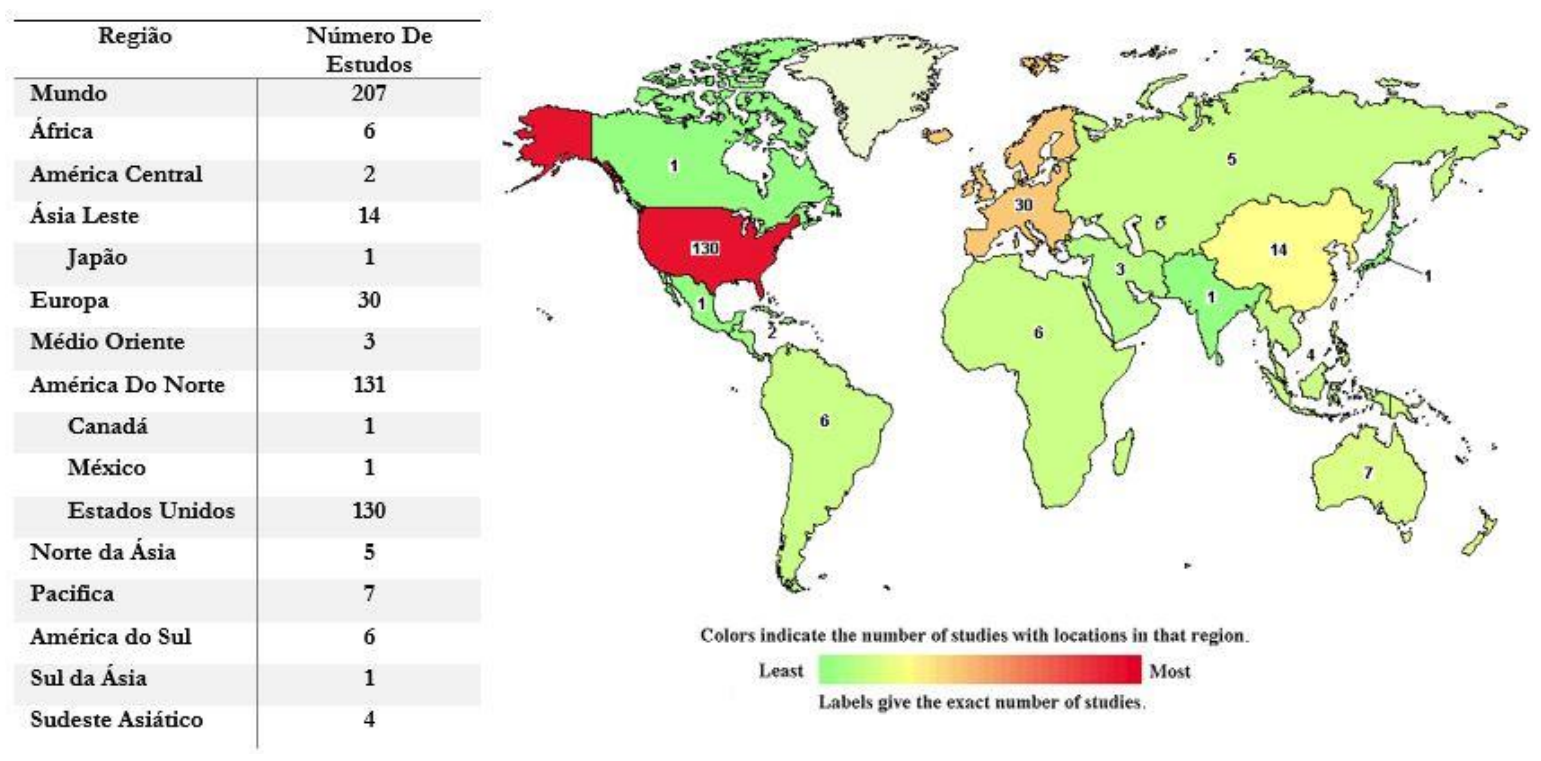

Fonte: CLINICALTRIALS.GOV (2018). 
Artigo original

Hegemonia - Revista Eletrônica do Programa de Mestrado em Direitos Humanos, Cidadania e Violência/Ciência Política do Centro Universitário Unieuro

ISSN: 1809-1261

UNIEURO, Brasília, número 25 (Especial), 2018, pp. 48-69.

De acordo com os dados das Figuras 2 a 4, para publicações de testes clínicos, a tendência ao aumento de publicações é observada. Verifica-se também esta tendência quando relacionado à patentes registradas no mundo. $\mathrm{Na}$ área da saúde, por exemplo, entre os anos de 2009 e 2012 houve um crescimento constante de 35\%, acompanhando o comportamento da área de nanotecnologia. E segundo a US Patent Collection (US PATENT COLLECTION, 2018), de 1976 até o presente (2 de maio de 2018), já foram publicadas 2010 patentes, em que em seu abstract continham os termos "nanoparticle" ou "nanostructure" e "health"ou "medicine" ou "treatment". A string utilizada foi (ABST/(nanoparticle OR nanostructure) AND ((health OR medicine) OR treatment)) .

\section{A PRODUÇÃO NANOTECNOLÓGICA DE ECRs TESTADAS EM HUMANOS NO BRASIL}

Dados citados na Figura 4 apresentam dados para pesquisas clínicas realizadas no Brasil. Estes trabalhos, foram realizados com produtos que possuíam o termo "nanoparticle OR nanostructure", contendo ou não resultados e relacionados a intervenções (testes clínicos), sem especificação de data. Os status das pesquisas foram: Ainda não recrutando, recrutando, se inscrevendo por convite, ativo, não recrutando e concluído. As pesquisas encontradas foram separadas em categoria de fármacos para: agentes anti-infecciosos, coagulantes, agentes dermatológicos, hipoglicemiantes, agentes reguladores de lipídios e agentes de controle reprodutivo. As regiões do Brasil em que se realizaram os testes foram em João Pessoa (Paraíba), Fortaleza (Ceará), Natal (RN), Diamantina (Minas Gerais) e São Paulo (São Paulo) (CLINICALTRIALS.GOV, 2018).

De acordo com o registro governamental de Ensaios Clínicos do Brasil (BRASIL, 2018), estão em andamento cerca de 1880 ECRs. Estes dados foram obtidos para pesquisas realizadas em:

- Redes de Pesquisas Clínicas, Agências Federais, Indústrias, Ministério da Saúde, Agências não Governamentais, Fundações Estaduais de Apoio à Pesquisa, Instituição de Ensino Superior, dentre outros;

- Com relação ao tipo interventional: em ambos os sexos;

- Pesquisas realizadas em ambos os sexos; e 
Artigo original

Hegemonia - Revista Eletrônica do Programa de Mestrado em Direitos Humanos, Cidadania e Violência/Ciência Política do Centro Universitário Unieuro

ISSN: 1809-1261

UNIEURO, Brasília, número 25 (Especial), 2018, pp. 48-69.

- Situação de recrutamento: variadas (desde aquelas ainda não recrutadas as com coleta de dados já concluídas).

Ainda com dados do site, registros de ensaios clínicos registrados nesta data (dado coletado em 13 de abril de 2018) são cerca de 5699 ensaios, sendo que 1867 foram publicados, 159 estão em análise e 637 em recrutamento. O draft total é de 3623.

Apesar do número expressivo de pesquisas em andamento, de acordo com as fontes, essas informações remetem que o Brasil se encontra em pleno desenvolvimento de estudos relacionados à nanotecnologia. Tal fato contribui para que este setor seja avaliado atenciosamente para que obtenha mais investimentos, consequentemente, e aproveite todo o potencial que o país possui.

AS NANOPARTÍCULAS E NANOESTRUTURAS MAIS PESQUISADAS NO BRASIL E NO MUNDO PARA APLICAÇÃO NA SAÚDE HUMANA

A nanotecnologia cada vez mais está conquistando o seu espaço dentro das pesquisas e do mercado comercial, uma vez que ela pode estar sendo inserida em qualquer área usual, como, saúde, agroindústria, energia, biotecnologia e etc. Dentro de cada exemplo, ela tem sua aplicação em função exponencial do que pode ser usada (SILVA, 2018).

Na saúde, como já citado, pode-se utilizá-la para tratamento de úlceras, cânceres, ressonância magnética, etc (SILVA, 2018). As pesquisas nos bancos de dados mostraram publicações contendo diversos estudos sobre tratamento de doenças, como por exemplo: diversos tipos de câncer, no Human Immunodeficiency Virus (HIV)/Síndrome da Imunodeficiência Adquirida ou Acquired Immunodeficiency Syndrome (AIDS), malária, Gastroenterologia etc. As pesquisas em nanomedicina são diretamente beneficiadas pelos avanços em biologia molecular e em nanorobótica. Atualmente decorrem muitos estudos sobre os efeitos de nanopartículas e nanorobôs dentro do corpo humano.

Segundo pesquisas na base de dados Web of Science, em abril de 2018, com o string: “Tópico:(nano*) AND Tópico: (biomedical) OR Tópico: (health) OR Tópico:(medicine) AND Tópico: (treatment) OR Tópico: (diagnosis) AND Tópico:("Clinical Trials" AND Randomized) ORTópico: (Trials AND "Randomized Clinical") OR Tópico: ("Controlled Clinical Trials" 
Artigo original

Hegemonia - Revista Eletrônica do Programa de Mestrado em Direitos Humanos, Cidadania e Violência/Ciência Política do Centro Universitário Unieuro

ISSN: 1809-1261

UNIEURO, Brasília, número 25 (Especial), 2018, pp. 48-69.

AND Randomized) OR Tópico: ("Randomized Clinical Trial")", apresentaram-se 1.324.918 trabalhos publicados nos últimos 10 anos. Quando selecionada apenas a categoria de “NANOSCIENCE NANOTECHNOLOGY” obtêm-se 10.680 trabalhos. Destes 9.257 são referentes a artigos e revisões. A Figura 5 mostra a quantidade de publicações, por ano, deste total, enquanto que a Figura 5 mostra os países/regiões envolvidos.

Figura 5. Total de publicações apresentadas na base Web of Science com string específico entre os anos jan/2008 e abril/2018, para a "Nanoscience Nanotechnology".

\section{Total de publicaçōes}

\subsection{7}

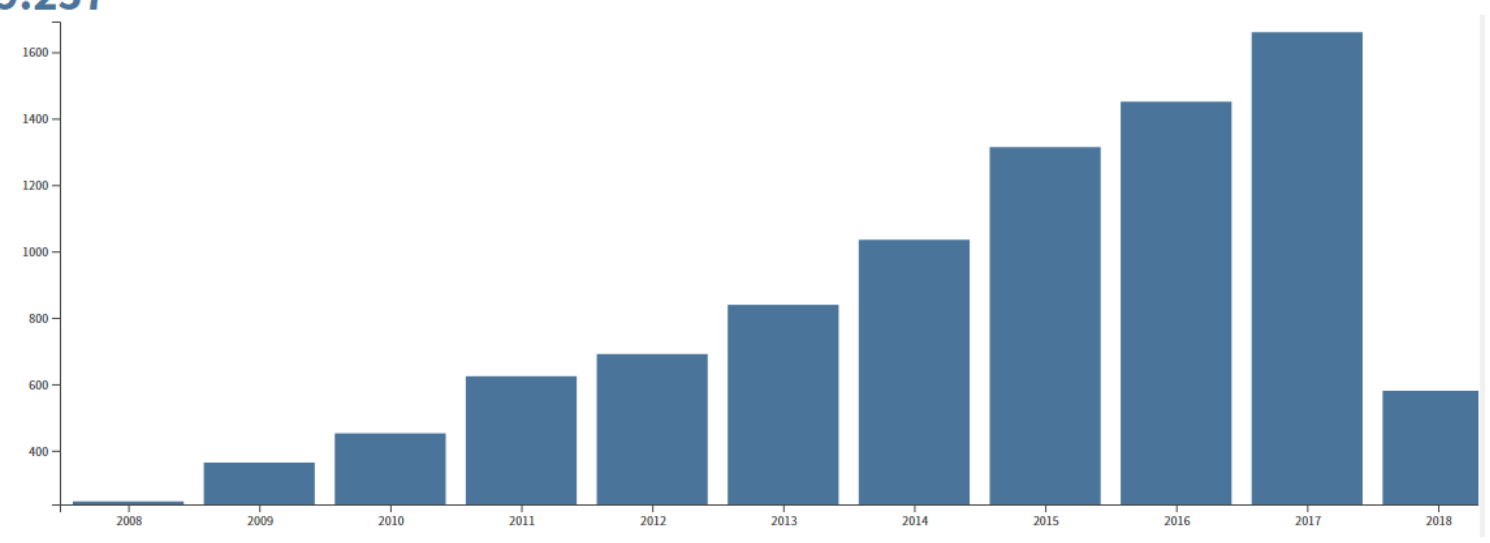

Fonte: Web of Science (2018). 
Artigo original

Hegemonia - Revista Eletrônica do Programa de Mestrado em Direitos Humanos, Cidadania e Violência/Ciência Política do Centro Universitário Unieuro

ISSN: 1809-1261

UNIEURO, Brasília, número 25 (Especial), 2018, pp. 48-69.

Figura 6. Países/Regiões nas quais as pesquisas foram realizadas para publicações dos trabalhos apresentados pela busca na Web of Science

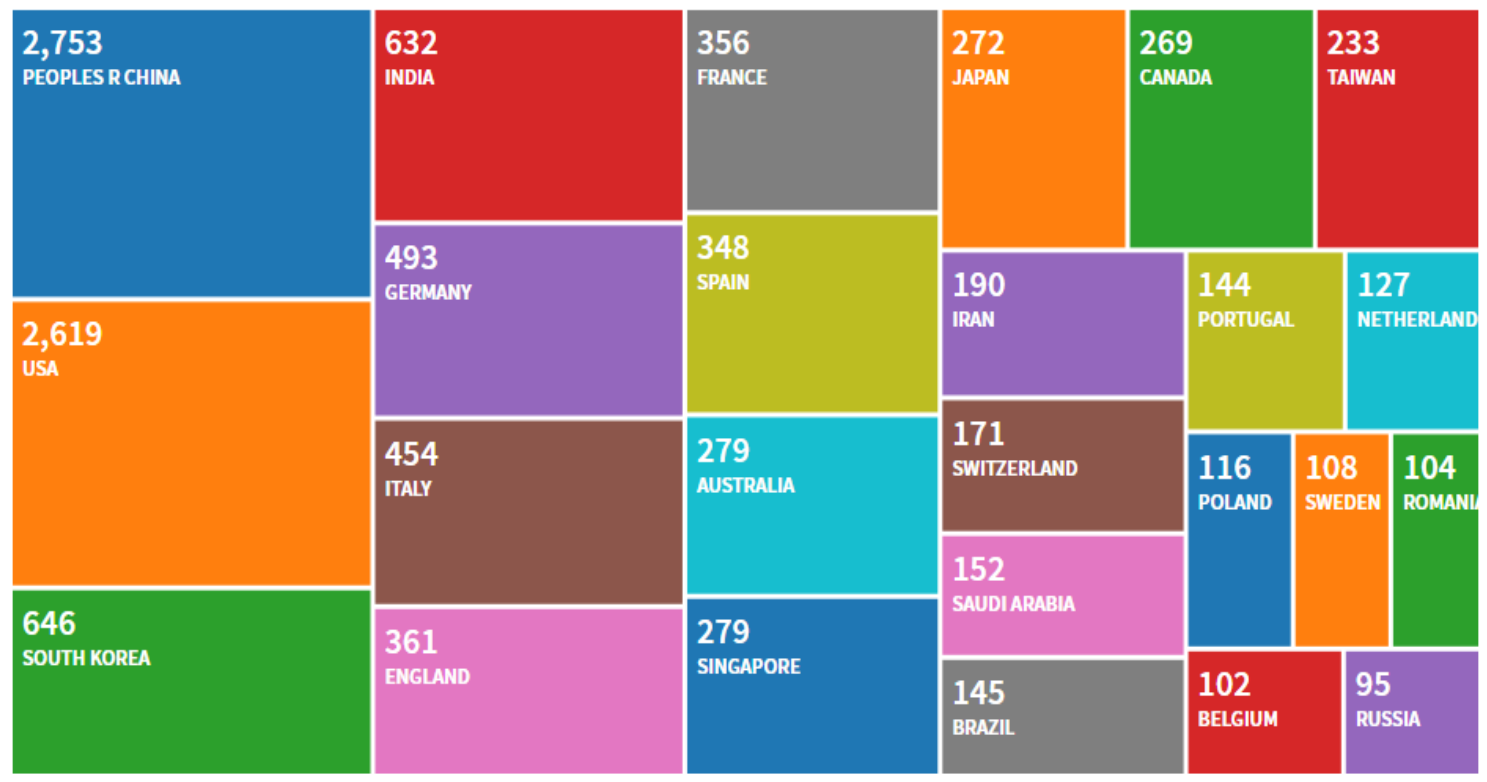

Fonte: Web of Science (2018).

Dos resultados das figuras 5 e 6, 2257 documentos são relacionados a "SCIENCE TECHNOLOGY OTHER TOPICS", 809 a "BIOTECHNOLOGY APPLIED MICROBIOLOGY", 439 a "PHARMACOLOGY PHARMACY", 281 a "RESEARCH EXPERIMENTAL MEDICINE", entre outros. A categoria "PHARMACOLOGY PHARMACY" tem como os maiores responsáveis pelas publicações, em ordem decrescente: China, Estados Unidos da América, Coréia do Sul, Índia e Itália. O Brasil é o $11^{\circ}$ que mais publicou nesta categoria, no período. Dentre os 439 trabalhos encontram-se recorrências e conectividade entre termos específicos, os quais são apresentados na Figura 7, que se segue. 
Artigo original

Hegemonia - Revista Eletrônica do Programa de Mestrado em Direitos Humanos, Cidadania e Violência/Ciência Política do Centro Universitário Unieuro

ISSN: $1809-1261$

UNIEURO, Brasília, número 25 (Especial), 2018, pp. 48-69.

Figura 7. Dentre os 439 trabalhos da categoria "PHARMACOLOGY PHARMACY" foram encontradas recorrências e conectividade entre termos específicos (Análise no software

VOSVIEWER 1.6.8 (2018))

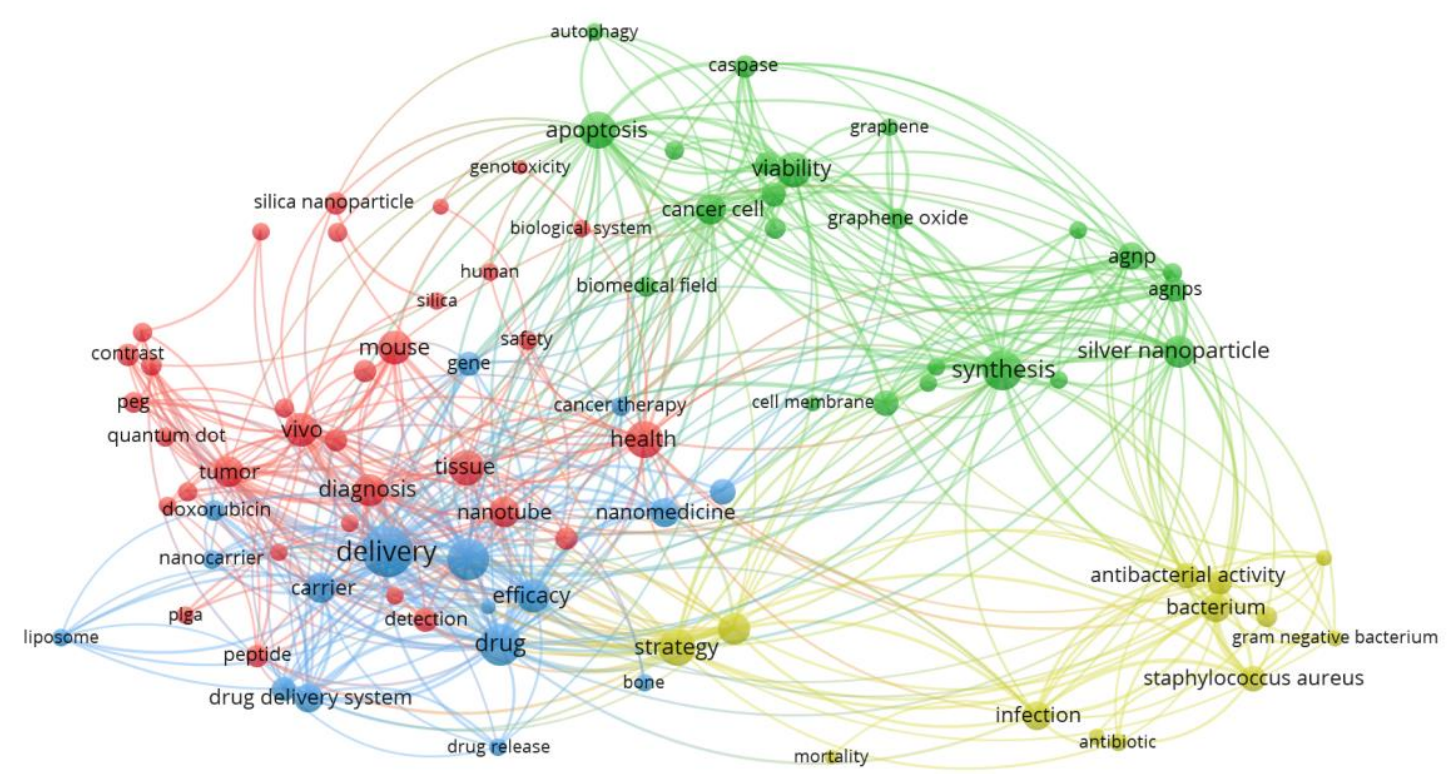

\& Vosviewer

Fonte: Web of Science (2018).

A Figura 7 mostra os termos mais utilizados nos artigos e revisões publicados entre 2008 e 2018, nos quais revelaram-se:

- Nanopartículas de sílica (LEE, S. et al., 2013; GUO, C. et al., 2015; YANG, H. et al., 2017);

- Nanopartículas de óxido de ferro (LLOYD-PARRY et al., 2018; MONDAL et al., 2017; SALIMI et al., 2018; UNTERWEGER et al., 2018; WU et al., 2017);

- Nanotubos de carbono (AL FARAJ; SHAIK; SHAIK, 2015; CHENG; CHENG, 2012; YAN et al., 2016);

- Grafeno (JANG et al., 2018; OU et al., 2017; YAN et al., 2016);

- Nanopartículas de prata (GURUNATHAN et al., 2015; YUAN et al., 2017);

- Quantum dots (HUANG et al., 2017; KIM et al., 2015; MADANI et al., 2013); 
Artigo original

Hegemonia - Revista Eletrônica do Programa de Mestrado em Direitos Humanos, Cidadania

e Violência/Ciência Política do Centro Universitário Unieuro

ISSN: $1809-1261$

UNIEURO, Brasília, número 25 (Especial), 2018, pp. 48-69.

- Lipossomas (BOZZUTO; MOLINARI, 2015; HAFNER et al., 2014; KNEIDL et al., 2014; LIU et al., 2017; SHEN et al., 2015; VIEIRA; GAMARRA, 2016; WEI et al., 2017; YAN et al., 2017),

- Entre outros.

De acordo com os achados, observa-se que as pesquisas com nanotecnologia e saúde têm se desenvolvido notavelmente, apesar das limitações tecnológicas que ainda persistem nas pesquisas científicas.

\section{CONCLUSÃO}

Tomando por base a bibliografia coletada durante as buscas, a nanotecnologia tem evoluído, significativamente, nos últimos anos no mundo, com destaque para China e Estados Unidos. No Brasil, as publicações de ECRs ainda são tímidas, segundo os registros de ensaios clínicos brasileiros e publicação de patentes. Além disso, os dados mostram que as pesquisas brasileiras foram realizadas, em sua maioria, na região nordeste do país.

Ainda sobre o resultado das buscas bibliográficas, os artigos publicados mostraram trabalhos focados em nanopartículas ou nanoestruturas como: nanopartículas de sílica, nanopartículas de óxido de ferro, nanotubos de carbono, grafeno, nanopartículas de prata, quantum dots, lipossomas, entre outros. Em geral, entre 2008 a 2017, o número de publicações sobre este tema cresceu em 76,57\%; e somente em 2018 a quantidade de publicações já passou de 400 .

Assim, é possível afirmar que as pesquisas com ECRs, aliados à nanotecnologia e saúde, estão avançando consideravelmente nos últimos dez anos. Seus produtos estão tomando o seu espaço nos mercados industriais e nos centros de pesquisas científicas, com aplicações nas mais diversas áreas do conhecimento. 
Artigo original

Hegemonia - Revista Eletrônica do Programa de Mestrado em Direitos Humanos, Cidadania e Violência/Ciência Política do Centro Universitário Unieuro

ISSN: $1809-1261$

UNIEURO, Brasília, número 25 (Especial), 2018, pp. 48-69.

\section{AGRADECIMENTOS}

Agradecemos à Universidade de Brasília-UnB e à Faculdade Gama-UnB/FGA pelo suporte na realização deste trabalho. Agradecemos também à CAPES pela disponibilização de bolsas de estudo e pesquisa.

\section{REFERÊNCIAS}

AL FARAJ, A.; SHAIK, A. P.; SHAIK, A. S. Magnetic single-walled carbon nanotubes as efficient drug delivery nanocarriers in breast cancer murine model: noninvasive monitoring using diffusion-weighted magnetic resonance imaging as sensitive imaging biomarker. International Journal of Nanomedicine, v. 10, p. 157-168, 2015.

ALBUQUERQUE, I. O. DE. Ensaio clínico para a avaliação da segurança e eficácia da terapia fotodinâmica mediada por nanoemulsão de al-cl-ftalocianina no tratamento do carcinoma basocelular de pele. 2017. 97 f., il. Tese (Doutorado em Nanociências e Nanobiotecnologia) Universidade de Brasília, Brasília, 2017.

BARBOSA, G. et al. Nanotechnology applied in drug delivery. In: IFMBE Proceedings-World Congress on Medical Physics and Biomedical Engineering. 51. ed. Toronto, Canadá: Springer International Publishing Switzerland 2015, 2015. p. 911-914.

BOZZUTO, G.; MOLINARI, A. Liposomes as nanomedical devices. International Journal of Nanomedicine, v. 10, p. 975-999, 2015.

BASTOS, R. M. DE P. Nanotecnologia: Uma Revolução no Desenvolvimento de Novos Produtos. 2006. 28 p. Juiz de Fora-MG: Trabalho de Conclusão de Curso - Universidade Federal de Juiz de Fora, Engenharia de Produção, Juiz de Fora, 2006..

BRASIL. Diretrizes metodológicas : elaboração de revisão sistemática e metanálise de ensaios clínicos randomizados. Brasília-DF: Ministério da Saúde, Secretaria de Ciência, Tecnologia e Insumos Estratégicos, Departamento de Ciência e Tecnologia, 2012.

BRASIL. Ministério da Saúde. Registro Brasileiro de Ensaios Clínicos. Disponível em: http://www.ensaiosclinicos.gov.br. Acesso em: 15 abr. 2018. 
Artigo original

Hegemonia - Revista Eletrônica do Programa de Mestrado em Direitos Humanos, Cidadania e Violência/Ciência Política do Centro Universitário Unieuro

ISSN: $1809-1261$

UNIEURO, Brasília, número 25 (Especial), 2018, pp. 48-69.

CHENG, J.; CHENG, S. H. Influence of carbon nanotube length on toxicity to zebrafish embryos. International Journal of Nanomedicine, v. 7, p. 3731-3739, 2012.

CLINICALTRIALS.GOV. ClinicalTrials.gov. Disponível em: $<$ https:/ / clinicaltrials.gov/ct2/results?cond $=\&$ term $=$ nanoparticle\&intr $=$ Drug\&strd_s $=01 \% 2$ F01\%2F2008\&strd_e=\&lupd_s=\&lupd_e=02\%2F05\%2F2018\&cntry $=\&$ state $=\&$ city $=\&$ dist $=$ $\&$ Search $=$ Search\&flds $=$ aby\&rslt $=$ With\&type $=$ Intr $>$. Acesso em: 2 maio. 2018.

COOK, D. J. et al. Rules of evidence and clinical recommendations on the use of antithrombotic agents. Chest, Northbrook, v. 102, n. 4, p. 305S-311S, 1992.

FERRO, A. F. P. Gestão da inovação aberta: práticas e competências em P\&D colaborativa. Tese (doutorado) Universidade Estadual de Campinas, Instituto de Geociências, 2010.

GARCIA, Renato. Internacionalização comercial e produtiva na indústria de cosméticos: desafios competitivos para empresas brasileiras. Revista Produção, v. 15, n. 2, p. 158-171, Maio/Ago. 2005

GUO, C. et al. Silica nanoparticles induce oxidative stress, inflammation, and endothelial dysfunction in vitro via activation of the MAPK/Nrf2 pathway and nuclear factor-kappa B signaling. International Journal of Nanomedicine, v. 10, p. 1463-1477, 2015.

GURUNATHAN, S. et al. Reduced graphene oxide-silver nanoparticle nanocomposite: a potential anticancer nanotherapy. International Journal of Nanomedicine, v. 10, p. 6257-6276, 2015.

HAFNER, A. et al. Nanotherapeutics in the EU: an overview on current state and future directions. International Journal of Nanomedicine, v. 9, p. 1005-1023, 2014.

HUANG, Y. et al. Current applications and future prospects of nanomaterials in tumor therapy. International Journal of Nanomedicine, v. 12, p. 1815-1825, 2017.

JANG, S.-C. et al. Nano-graphene oxide composite for in vivo imaging. International Journal of Nanomedicine, v. 13, p. 221-234, 2018.

KIM, J.-H. et al. Carcinogenic activity of PbS quantum dots screened using exosomal biomarkers secreted from HEK293 cells. International Journal of Nanomedicine, v. 10, p. 5513-5528, 2015. 
Artigo original

Hegemonia - Revista Eletrônica do Programa de Mestrado em Direitos Humanos, Cidadania e Violência/Ciência Política do Centro Universitário Unieuro

ISSN: 1809-1261

UNIEURO, Brasília, número 25 (Especial), 2018, pp. 48-69.

KNEIDL, B. et al. Thermosensitive liposomal drug delivery systems: state of the art review. International Journal of Nanomedicine, v. 9, p. 4387-4398, 2014.

LEE, S. et al. The comparative immunotoxicity of mesoporous silica nanoparticles and colloidal silica nanoparticles in mice. International Journal of Nanomedicine, v. 8, p. 147-158, 2013.

LEITÃO, E. C. DE V. Terapia fotodinâmica mediada por cloreto alumínio-ftalocianina em nanoemulsões para tratamento de pacientes com queilite actínica : análise clínica e histopatológica. Tese de doutorado. Universidade de Brasília, Instituto de Ciências Biológicas, Programa de Pós-Graduação em Nanociência e Nanobiotecnologia, 29 maio 2017.

LIU, C. et al. A dual-mediated liposomal drug delivery system targeting the brain: rational construction, integrity evaluation across the blood-brain barrier, and the transporting mechanism to glioma cells. International Journal of Nanomedicine, v. 12, p. 2407-2425, 2017.

LLOYD-PARRY, O. et al. Nanomedicine applications in women's health: state of the art. International Journal of Nanomedicine, v. 13, p. 1963-1983, 2018.

LUZ, G. V. DA S. et al. Nanorobotics in Drug Delivery Systems for Treatment of Cancer: A Review. Journal of Materials Science and Engineering A, v. 6, n. 3, 2016.

MADANI, S. Y. et al. Conjugation of quantum dots on carbon nanotubes for medical diagnosis and treatment. International Journal of Nanomedicine, v. 8, p. 941-950, 2013.

MALAVOLTA, E. A. et al. Ensaios clínicos controlados e randomizados na ortopedia: dificuldades e limitações. Revista Brasileira de Ortopedia, v. 46, n. 4, p. 452-459, 2011.

MONDAL, S. et al. Magnetic hydroxyapatite: a promising multifunctional platform for nanomedicine application. International Journal of Nanomedicine, v. 12, p. 8389-8410, 2017.

NNI - National Nanotechnology Initiative. U.S. Government. Disponível em: <https://www.nano.gov/>. Acesso em: 28 abr. 2018.

OU, L. et al. The mechanisms of graphene-based materials-induced programmed cell death: a review of apoptosis, autophagy, and programmed necrosis. International Journal of Nanomedicine, v. 12, p. 6633-6646, 2017. 
Artigo original

Hegemonia - Revista Eletrônica do Programa de Mestrado em Direitos Humanos, Cidadania e Violência/Ciência Política do Centro Universitário Unieuro

ISSN: 1809-1261

UNIEURO, Brasília, número 25 (Especial), 2018, pp. 48-69.

SALIMI, M. et al. Biodistribution, pharmacokinetics, and toxicity of dendrimer-coated iron oxide nanoparticles in BALB/c mice. International Journal of Nanomedicine, v. 13, p. 1483 1493, 2018.

SAHA, M. Nanomedicine: Promising Tiny Machine for the Healthcare in Future-A Review. Oman Medical Journal, v. 24, n. 4, p. 242-7, out. 2009.

SILVA, M. M. P. et al. Utilização de nanopartículas no tratamento de feridas: revisão sistemática. Revista da Escola de Enfermagem da USP, v. 51, n. 0, 8 jan. 2018.

SOUZA, R. F. O que é um estudo clínico randomizado? Medicina, v. 42, n. 1, p. 3-8, 2009.

SCHAFFAZICK, S. R.; FREITAS, L. L.; POHLMANN, A. R.; GUTERRES, S. S. (2003); "Caracterização e estabilidade físico-química de sistemas poliméricos nanoparticulados para administração de fármacos”. Química Nova, v. 25, n. 5, p. 726-737.

SHEN, S. et al. Compound antimalarial ethosomal cataplasm: preparation, evaluation, and mechanism of penetration enhancement. International Journal of Nanomedicine, v. 10, p. 4239_ 4253, 2015.

UNTERWEGER, H. et al. Dextran-coated superparamagnetic iron oxide nanoparticles for magnetic resonance imaging: evaluation of size-dependent imaging properties, storage stability and safety. International Journal of Nanomedicine, v. 13, p. 1899-1915, 2018.

US PATENT COLLECTION. Patent Database Search Results. Disponível em: $<$ http://patft.uspto.gov/netacgi/nph-

Parser?Sect $1=$ PTO $2 \&$ Sect $2=$ HITOFF\&u= $\% 2$ Fnetahtml $\% 2$ FPTO $\% 2$ Fsearch adv.htm\&r=0\&f=S\&l=50\&d=PTXT \&RS=ABST $\% 2 \mathrm{~F} \% 28$ nanoparticle $+\mathrm{OR}+$ nanostructure $\% 29 \&$ Refine $=$ Refine + Search $\&$ Query $=$ ABST $\% 2 F \% 28$ nanoparticle $+\mathrm{OR}+$ nanostructure $\% 29+$ AND+\%28health+OR>. Acesso em: 2 maio. 2018.

VIEIRA, D. B.; GAMARRA, L. F. Getting into the brain: liposome-based strategies for effective drug delivery across the blood-brain barrier. International Journal of Nanomedicine, v. 11, p. 5381-5414, 2016.

WEI, Y. et al. Lung-targeting drug delivery system of baicalin-loaded nanoliposomes: development, biodistribution in rabbits, and pharmacodynamics in nude mice bearing orthotopic human lung cancer. International Journal of Nanomedicine, v. 12, p. 251-261, 2017. 
Artigo original

Hegemonia - Revista Eletrônica do Programa de Mestrado em Direitos Humanos, Cidadania e Violência/Ciência Política do Centro Universitário Unieuro

ISSN: 1809-1261

UNIEURO, Brasília, número 25 (Especial), 2018, pp. 48-69.

WILLEMS \& VAN DEN WILDENBERG. "NanoRoadMap: Work document on Nanomaterials".W\&W España s.l. 2004.

WU, Q. et al. Iron oxide nanoparticles and induced autophagy in human monocytes. International Journal of Nanomedicine, v. 12, p. 3993-4005, 2017.

YAN, M. et al. Systemic toxicity induced by aggregated layered double hydroxide nanoparticles. International Journal of Nanomedicine, v. 12, p. 7183-7195, 2017.

YAN, X. et al. Graphene/single-walled carbon nanotube hybrids promoting osteogenic differentiation of mesenchymal stem cells by activating p38 signaling pathway. International Journal of Nanomedicine, v. 11, p. 5473-5484, 2016.

YANG, H. et al. Biodegradable mesoporous delivery system for biomineralization precursors. International Journal of Nanomedicine, v. 12, p. 839-854, 2017.

YUAN, Y.-G. et al. Quercetin-mediated synthesis of graphene oxide-silver nanoparticle nanocomposites: a suitable alternative nanotherapy for neuroblastoma. International Journal of Nanomedicine, v. 12, p. 5819-5839, 2017. 